\title{
A review of interventional treatments for colorectal lung metastases: is it time for a change in practice?
}

\author{
Marcus Taylor, Udo Abah, Rajesh Shah \\ Department of Cardiothoracic Surgery, Wythenshawe Hospital, Manchester University NHS Foundation Trust, Manchester, M23 9LT, UK
}

Correspondence to: Marcus Taylor. Department of Cardiothoracic Surgery, Wythenshawe Hospital, Manchester University NHS Foundation Trust, Southmoor Road, Manchester, M23 9LT, UK. Email: Marcus.taylor1@nhs.net.

Provenance and Peer Review: This article was commissioned by the Editorial office, Quantitative Imaging in Medicine and Surgery. The article did not undergo external peer review.

Comment on: Hasegawa T, Takaki H, Kodama H, Yamanaka T, Nakatsuka A, Sato Y, Takao M, Katayama Y, Fukai I, Kato T, Tokui T, Tempaku H, Adachi K, Matsushima Y, Inaba Y, Yamakado K. Three-year Survival Rate after Radiofrequency Ablation for Surgically Resectable Colorectal Lung Metastases: A prospective multicenter study. Radiology 2020;294:686-95.

Submitted Apr 14, 2020. Accepted for publication Apr 26, 2020.

doi: 10.21037/qims-2020-15

View this article at: http://dx.doi.org/10.21037/qims-2020-15

\section{Background}

Colorectal cancer is the third most frequently occurring malignancy across the globe, with over 1.8 million new diagnoses in 2018 alone. Approximately one-fifth of patients have evidence of metastasis at the time of initial diagnosis and five-year survival for untreated stage IV colorectal cancer is reported to be as low as $10 \%$ (1). A number of studies have shown that patients with isolated metastases receiving radical treatment can have 5-year survival rates of up to $50 \%$ (2). The term "metastatic colorectal cancer" covers a diverse spectrum of pathology, with treatment strategies varying greatly, dependent upon the extent and site of metastasis. Common sites of spread include liver, lung, bone, brain and peritoneum, with the lungs the second most common location after the liver. Metastases are termed to be either 'synchronous' or 'metachronous', dependent upon whether they are identified during the initial diagnostic work-up of the primary colorectal tumour (synchronous) or at any point afterwards(metachronous). 'Initial' (as opposed to 'non-initial') is a term used to delineate the sequence of metastatic spread. The term 'initial lung metastasis' refers to a specific situation where the lungs are the first site of metastasis. An additional classification addresses whether the lung metastasis is 'isolated' (not accompanied by additional extrapulmonary metastasis) or 'non-isolated'. Presence or absence of either the original primary tumour or a local recurrence of this primary tumour does not affect the classification of lung metastases as either isolated or non-isolated (3).

\section{Evidence base for pulmonary metastasectomy}

Traditionally, surgical resection of lung metastases has been recognised as the most effective treatment with regards to extending overall survival, despite a lack of highquality evidence to support this theory. However, some experts have challenged this practice and the topic remains contentious amongst thoracic surgeons. Whilst a number of retrospective studies demonstrated significantly longer disease-free and overall survival for patients undergoing lung resection in comparison to those patients who did not, it is difficult to avoid selection bias in studies of this type (4). Despite identifying over 50 papers, a 2010 systematic review was unable to draw any robust conclusions as to the effect of surgical metastasectomy, based on the poor quality of the evidence identified (5). An additional systematic review from 2013, which included 25 studies, was also unable to either confirm or refute the benefit of surgical resection, as all studies were single-arm and no controlled trials had been 
undertaken at the time of review (6). The 5 -year survival rates ranged from $27 \%$ to $68 \%$ in the studies included in the review. In a further literature review undertaken by Pfannschmidt et al. (2), 5-year survival rates ranged from $32.7 \%$ to $56 \%$.

In 2019 a randomised controlled trial was published which aimed to address this controversial issue. The Pulmonary Metastasectomy versus Continued Active Monitoring in Colorectal Cancer (PulMiCC) trial was a multi-centre study which randomised 65 patients to either surgical metastasectomy or continued active clinical monitoring. Problems with recruitment, as demonstrated by the relatively small number of patients included, left the study underpowered to draw meaningful conclusions, a fact acknowledged by the trial group. Whilst no significant difference in 5-year survival was demonstrated between the metastasectomy and control arms of the PulMiCC trial (38\% vs. $29 \%$ respectively, HR 0.82 , 95\% CI: $0.43-1.56$ ), the authors felt that the most important outcome of the study was the 5 -year survival rate of $29 \%$ (16-52\%) for those patients in the control arm. This is a substantially higher 5 -year survival rate compared to what has previously been reported in the literature and alters the context in which surgical metastasectomy is currently considered.

We agree with the authors of the PulMiCC trial, who state that (although non-significant), a HR of 0.82 suggests that it is likely that in some patients, for whom isolated lung metastasis remains the only remnant of their otherwise fully-treated colorectal cancer, pulmonary metastasectomy is likely to convey benefit. However the study also strongly suggests that the previously-held belief that similar patients who do not undergo surgical resection have a very small chance of surviving to 5 years should be re-examined (7).

\section{Criteria for surgical metastasectomy}

Despite the absence of formal criteria, within the literature there is broad consensus regarding inclusion and exclusion criteria for patients to be deemed suitable to undergo surgical metastasectomy. As with any thoracic surgical procedure, patients must possess an appropriate degree of physiological reserve to withstand the procedure and should not be at a prohibitively high risk of perioperative mortality or post-operative dyspnoea. From an anatomical perspective, complete resection of all pulmonary metastases should be anatomically achievable. Oncological considerations include ensuring that the primary tumour has been appropriately treated (either fully resected or under therapeutic control). Decisions regarding treatment should be made by a multidisciplinary team on an individual case by case basis, particularly in the presence of more complex factors such as the presence of extrapulmonary disease. Interval imaging to demonstrate metastatic disease stability is also felt to be of importance when determining patient suitability for treatment (4).

\section{Alternative treatment modalities}

When considering these criteria for patient selection it is understandable that a substantial proportion of patients with lung metastases will be deemed unsuitable for surgical resection. For these patients, alternative treatment modalities such as chemotherapy, stereotactic ablative body radiotherapy (SABR) and radiofrequency ablation (RFA) can be considered. However, as with surgical treatment, high quality data assessing these treatments is either severely lacking or absent altogether. A large retrospective study published in 2017 demonstrated a median overall survival of 23.8 months for patients with initial lung metastases who received treatment with chemotherapy (8), which is similar to the findings in other published studies (9). SABR delivers high energy radiation via high-precision external beams and has been used more frequently in recent times in the management of primary pulmonary tumours as first-line treatment for those patients who are unsuitable for surgical resection. A 2017 retrospective review of 44 patients with isolated lung metastases treated with SABR demonstrated an overall median survival of 38 months and 3-year survival of $50.8 \%$ (10). However, SABR is associated with a number of serious complications, particularly radiation pneumonitis, which has been reported to be as high as $8 \%$ in certain studies (9).

\section{Radiofrequency ablation}

RFA is a minimally invasive percutaneous technique which does not necessarily need to be performed under general anaesthesia. A high-frequency electrical current is delivered through an electrode. This current heats the area of lung parenchyma around the electrode to a temperature in excess of $60^{\circ} \mathrm{C}$ and subsequently causes focal necrosis of tumour tissue. In the context of treating lung metastases, studies have shown that the technique is more effective when metastases are small $(<3 \mathrm{~cm}$, although one study showed a further improvement in outcomes when using $<2 \mathrm{~cm}$ as the cut-off point) and when no more than three metastases are 
present (11).

Benefits of the technique include a very low mortality rate (quoted as $0-0.4 \%$ in previous studies) and the minimally invasive aspect of the procedure. Nevertheless, there are also a number of associated drawbacks such as a fairly high rate of procedural morbidity. Complications include pneumothorax (occurring in between one-third and two-thirds of all cases, although the number of patients requiring a chest drain is substantially lower), bleeding, haemoptysis, pleural effusion and infection (4). The other main drawback associated with RFA is the lack of an unequivocal histological diagnosis [for those patients who have not had a biopsy of the lesion(s) prior to RFA], as the nature of the treatment means that post-procedural histological analysis cannot be undertaken, as it would be after surgical removal of a lesion.

Several studies assessing the efficacy of RFA in treating colorectal lung metastases have been published, including a 2013 systematic review which included three studies (12). These studies reported a 3-year survival rate of 46-60\% and a 5-year survival rate of 34.9-45\%. All three studies were retrospective, and all contained only small numbers of patients ( $\mathrm{n}=78,55$ and 102). A larger study from 2015 reviewed the outcomes of 566 patients who underwent RFA in 2 French centres between 2002 and 2010 with a median follow-up of 35.5 months (11). The average number of metastases per patient was 1.8 and the median diameter of metastases was $15 \mathrm{~mm}$. The 3-year survival rate was $67.7 \%$ and the 5 -year survival rate was $51.5 \%$. Patients received the treatment under general anaesthesia and post-procedural complication rates were similar to other published experiences. Pneumothorax occurred in $67 \%$ of cases, of which just over half required treatment with a chest drain.

Hasegawa et al. have published their multicentre experience of patients undergoing RFA in 6 centres across Japan between 2008 and 2014 (13). This is the first prospective study undertaken to assess the efficacy of RFA in the management of colorectal lung metastases. The study recruited seventy patients with a total of 100 lesions which were all deemed anatomically resectable as well as suitable to undergo RFA. Mean lesion diameter was $1.0 \mathrm{~cm}(\mathrm{SD} \pm 0.5 \mathrm{~cm}$, range, $0.4-2.8 \mathrm{~cm})$ and $30 \%$ of patients $(n=21)$ had more than one lesion. Inclusion criteria included previous complete resection of the primary tumour with no evidence of locoregional recurrence and no evidence of extrapulmonary metastasis, meaning that this study was designed solely to assess those patients with isolated metachronous colorectal lung metastases. Additional inclusion criteria included a maximum lesion diameter of $3 \mathrm{~cm}$ and no more than five lesions present. These criteria correlate with previous studies, which have demonstrated that outcomes are better in patients with both smaller and fewer lesions.

However, patients who had undergone previous treatment for colorectal metastases, such as chemotherapy or previous metastasectomy, were not excluded from the trial. Indeed, almost one-third of patients $(n=22)$ had previously undergone surgical metastasectomy and one-fifth $(n=14)$ had previously undergone adjuvant chemotherapy. A further $21 \%$ of patients received chemotherapy after undergoing RFA. Moreover, although the presence of extrapulmonary metastasis was a specific exclusion criterion, it is not clear whether patients with previously resected extrapulmonary metastasis (i.e., previous liver resection for isolated liver metastasis) were included in the study.

The other major drawback with this study was the large proportion of patients $(93 \%, \mathrm{n}=65)$ who didn't have histological confirmation of the presence of colorectal lung metastasis prior to undergoing RFA. Lesions were considered to be pathological if they had increased by $30 \%$ or more on 3-monthly computed tomography (CT) scans, and/or if they showed positive uptake on positron emission tomography (PET) scan, although no information was given as to where the cut-off lay for differentiation between positive and negative uptake on PET scan. Biopsy was only performed if there was no PET uptake or if there were radiological features suggestive of primary lung cancer. Although concordance between PET and histology is generally accepted as being higher than the concordance between CT and histology, studies report this concordance as being around $70 \%$ (4), meaning that a substantial subgroup of patients may have undergone RFA of a benign nodule which was erroneously assumed to be malignant. Clearly this would have implications on both disease-free and overall survival rates.

Despite these drawbacks, Hasegawa et al. have published excellent results. The study demonstrated a 3-year survival rate (the designated primary endpoint of the study) of $84 \%$ with a mean follow-up of 57 months ( \pm 32 months, range, 0-115 months). After multivariable analysis rectal cancer, positive carcinoembryonic antigen (CEA) and an absence of previous chemotherapy were found to be independently associated with a worse overall prognosis. Three patients required a second session of RFA when the lesions were not fully ablated during the first session. Hence the authors 
Table 1 Published outcomes for patients receiving different treatment modalities for colorectal lung metastases

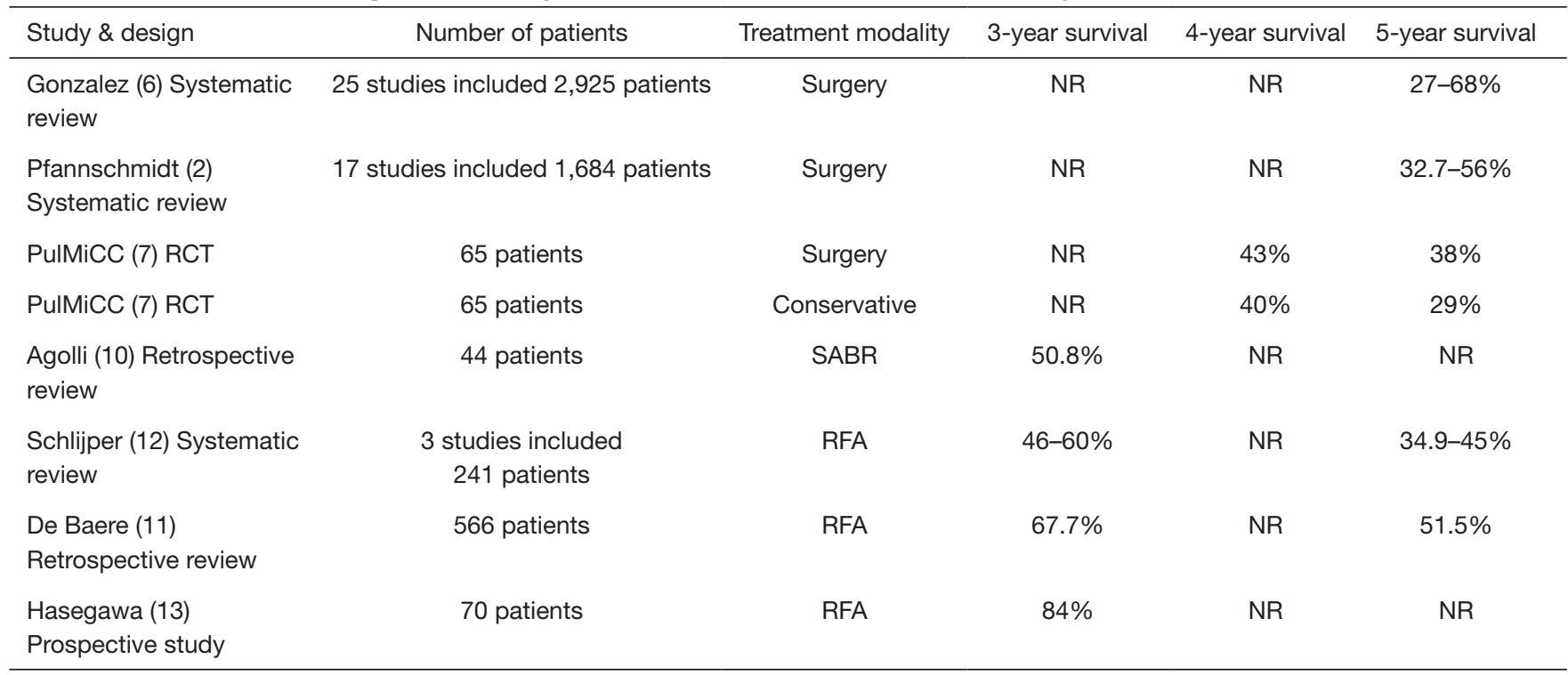

NR, not recorded; SABR, stereotactic ablative body radiotherapy; RFA, radiofrequency ablation.

report a primary technical success rate of $96 \%$. 30-day mortality was $1.43 \%(\mathrm{n}=1)$, due to one patient who died on day one post-procedure as a result of a large haemothorax. $43 \%(\mathrm{n}=30)$ of patients developed a pneumothorax, of which $60 \%(n=18)$ required treatment with a chest drain. These results demonstrate an excellent standard of technical efficacy and safety and are summarised in Table 1, alongside results from other published studies looking at outcomes after different treatment modalities.

\section{Conclusion}

The management of colorectal lung metastases remains controversial. Whilst a number of authors have examined the prognostic benefit of various therapies including surgery, radiotherapy and percutaneous ablation, no studies have been powered to show a statistically significant impact, and opponents of the intervention continue to suggest that the improved prognosis demonstrated in studies is due to positive selection bias. Hasegawa et al. have demonstrated that RFA is a safe and effective option for treating colorectal lung metastases, although a number of study limitations mean that these results should be interpreted with some caution. Whilst their 3-year overall survival rate is the highest published to date for any treatment modality for this patient population, only $7 \%$ of patients had pathological confirmation of diagnosis prior to treatment. Nevertheless, when compared to the results of the PulMiCC trial, (and indeed to all other published studies in this area), the 3-year results from Hasegawa et al. appear promising and we await the 5-year results with interest.

In conclusion, there remains very little high-quality evidence demonstrating the benefit of any form of intervention in this patient group and hence a controlled trial comparing overall survival between patients undergoing surgical metastasectomy, SABR or RFA for biopsy-proven metastatic colorectal cancer is required to address the question of which modality provides superior outcomes in this cohort of patients.

\section{Acknowledgments}

Funding: None.

\section{Footnote}

Conflicts of Interest: All authors have completed the ICMJE uniform disclosure form (available at: http://dx.doi. org/10.21037/qims-2020-15). The authors have no conflicts of interest to declare.

Open Access Statement: This is an Open Access article distributed in accordance with the Creative Commons Attribution-NonCommercial-NoDerivs 4.0 International 
License (CC BY-NC-ND 4.0), which permits the noncommercial replication and distribution of the article with the strict proviso that no changes or edits are made and the original work is properly cited (including links to both the formal publication through the relevant DOI and the license). See: https://creativecommons.org/licenses/by-nc-nd/4.0/.

\section{References}

1. Villeneuve PJ, Sundaresan RS. Surgical management of colorectal lung metastasis. Clin Colon Rectal Surg 2009;22:233-41.

2. Pfannschmidt J, Dienemann H, Hoffmann H. Surgical Resection of Pulmonary Metastases From Colorectal Cancer: A Systematic Review of Published Series. Ann Thorac Surg 2007;84:324-38.

3. Li J, Yuan Y, Yang F, Wang Y, Zhu X, Wang Z, Zheng S, Wan D, He J, Wang J, Ba Y, Bai C, Bai L, Bai W, Bi F, Cai K, Cai M, Cai S, Chen G, Chen K, Chen L, Chen P, Chi P, Dai G, Deng Y, Ding K, Fan Q, Fang W, Fang X, Feng F, Fu C, Fu Q, Gu Y, He Y, Jia B, Jiang K, Lai M, Lan P, Li E, Li D, Li J, Li L, Li M, Li S, Li Y, Li Y, Li Z, Liang X, Liang Z, Lin F, Lin G, Liu H, Liu J, Liu T, Liu Y, Pan H, Pan Z, Pei H, Qiu M, Qu X, Ren L, Shen Z, Sheng W, Song C, Song L, Sun J, Sun L, Sun Y, Tang Y, Tao M, Wang C, Wang H, Wang J, Wang S, Wang X, Wang X, Wang Z, Wu A, Wu N, Xia L, Xiao Y, Xing B, Xiong B, Xu J, Xu J, Xu N, Xu R, Xu Z, Yang Y, Yao H, Ye Y, Yu Y, Yu Y, Yue J, Zhang J, Zhang J, Zhang S, Zhang W, Zhang Y, Zhang Z, Zhang Z, Zhao L, Zhao R, Zhou F, Zhou J, Jin J, Gu J, Shen L. Expert consensus on multidisciplinary therapy of colorectal cancer with lung metastases (2019 edition). J Hematol Oncol 2019;12:16.

4. Moorcraft SY, Ladas G, Bowcock A, Chau I. Management of resectable colorectal lung metastases. Clin Exp Metastasis 2016;33:285-96.

5. Fiorentino F, Hunt I, Teoh K, Treasure T, Utley M. Pulmonary metastasectomy in colorectal cancer: A systematic review and quantitative synthesis. J R Soc Med 2010;103:60-6.

6. Gonzalez M, Poncet A, Combescure C, Robert J, Ris HB, Gervaz P. Risk factors for survival after lung

Cite this article as: Taylor M, Abah U, Shah R. A review of interventional treatments for colorectal lung metastases: is it time for a change in practice? Quant Imaging Med Surg 2020;10(6):1413-1417. doi: 10.21037/qims-2020-15 metastasectomy in colorectal cancer patients: A systematic review and meta-analysis. Ann Surg Oncol 2013;20:572-9.

7. T reasure T, Farewell V, Macbeth F, Monson K, Williams NR, Brew-Graves C, Lees B, Grigg O, Fallowfield L; PulMiCC Trial Group. Pulmonary Metastasectomy versus Continued Active Monitoring in Colorectal Cancer (PulMiCC): A multicentre randomised clinical trial. Trials 2019;20:718,

8. Wang Z, Wang X, Yuan J, Zhang X, Zhou J, Lu M, Liu D, Li J, Shen L. Survival Benefit of Palliative Local Treatments and Efficacy of Different Pharmacotherapies in Colorectal Cancer With Lung Metastasis: Results From a Large Retrospective Study. Clin Colorectal Cancer 2018;17:e233-55.

9. Ibrahim T, Tselikas L, Yazbeck C, Kattan J. Systemic Versus Local Therapies for Colorectal Cancer Pulmonary Metastasis: What to Choose and When? J Gastrointest Cancer 2016;47:223-31.

10. Agolli L, Bracci S, Nicosia L, Valeriani M, De Sanctis V, Osti MF. Lung Metastases Treated With Stereotactic Ablative Radiation Therapy in Oligometastatic Colorectal Cancer Patients: Outcomes and Prognostic Factors After Long-Term Follow-Up. Clin Colorectal Cancer 2017;16:58-64.

11. de Baère T, Aupérin A, Deschamps F, Chevallier $P$, Gaubert Y, Boige V, Fonck M, Escudier B, Palussiére J. Radiofrequency ablation is a valid treatment option for lung metastases: experience in 566 patients with 1037 metastases. Ann Oncol 2015;26:987-91.

12. Schlijper RCJ, Grutters JPC, Houben R, Dingemans AM, Wildberger JE, Van Raemdonck D, Van Cutsem E, Haustermans K, Lammering G, Lambin P, De Ruysscher D. What to choose as radical local treatment for lung metastases from colo-rectal cancer: Surgery or radiofrequency ablation? Cancer Treat Rev 2014;40:60-7.

13. Hasegawa T, Takaki H, Kodama H, Yamanaka T, Nakatsuka A, Sato Y, Takao M, Katayama Y, Fukai I, Kato T, Tokui T, Tempaku H, Adachi K, Matsushima Y, Inaba Y, Yamakado K. Three-year Survival Rate after Radiofrequency Ablation for Surgically Resectable Colorectal Lung Metastases: A prospective multicenter study. Radiology 2020;294:686-95. 